\title{
XXXIII. An attempt to determine whether a minimum time is necessary to excite the human retina
}

\section{J.H.J. Poole Sc.D.}

To cite this article: J.H.J. Poole Sc.D. (1922) XXXIII. An attempt to determine whether a minimum time is necessary to excite the human retina, Philosophical Magazine Series 6 , 43:254, 345-348, DOI: $10.1080 / 14786442208565221$

To link to this article: http://dx.doi.org/10.1080/14786442208565221

曲 Published online: 08 Apr 2009.

Submit your article to this journal $[\pi$

Џll Article views: 3

Q View related articles $\longleftarrow$

Citing articles: 3 View citing articles $\square$ 
XXXIII. An Attempt to determine whether a Minimum Time is necessary to excite the Human Retina. By J. H. J. POOLE, SC.D.**

$\mathbf{I}^{\mathrm{N}}$

a letter to 'Nature,' published on April 7th, 1921, Sir Oliver Lodge suggested that possibly there might be a certain minimum time necessary tor a beam of light to liberate an electron from a photo-electric surface. Thus, assuming that the area of wave-front which can contribute energy to a very small resonator, like the electron, is of the order of $\lambda^{2} / \pi$, he showed that for ordinary sunlight the surface would have to be illuminated for about $\frac{1}{1000}$ sec. before an electron could be liberated. It seems, however, improbable that such a comparatively large interval of time should be necessary to excite an electrun, especially as photographs, in which the action of the light on the sensitive film is probably due to the liberation of such electrons, can be taken in ordinary sunlight with exposures shorter than this.

As a matter of fact, at the time that Sir Olivor Lodge published the above suggestion in 'Nature,' Dr. Joly and I had already undertaken some experiments with the view to seeing if any such effect held for the human retina. The general principle of the method is described by Dr. Joly in a letter to 'Nature' for April 14th, 1921, but perhaps a recapitulation of it is desirable.

The general idea of the method was to cause a parallel beam of light to revolve very rapidly by reflecting it from the surface of a rotating mirror. The revolving beam was then viewed through a narrow slit placed at a considerable distance from the mirror ; and it is obvinus that if the slit and the light-beam can both be kept narrow, then the resulting flash, seen by the eye, may be made very short indeed by running the mirror at a high speed, and also by making the distance between the mirror and the slit large.

In order to get as high a speed of rotation of the mirror as possible, it was mounted on the top of the vertical spindle of a Legendre Centrifuge. This centrifuge was capable of speeds up to about 8000 r.p.m., as was determined with a revolution counter and stop-wateh. The mirror itself was a small speculum metal one, of about $4 \mathrm{cms}$. diameter, manufactured and ground optically plane by Sir Howard Grubb. During the course of the experiments this mirror

* Communicated by Prof. J. Joly, F.R.S. 
was re-polished by Hilger Ltd., as it was found that there was rather an undue amount of irregular reflexion taking place at the surface of the mirror. To avoid, as far as possible, any stray light from the mounting of the mirror or from its back reaching the eye, they were both cartiully blackened by means of a small turpentine flame, which gave a very gond derosit of lamp-black.

The chief difficulty experienced was with the optical arrangements. It is plain that it is impossible to realize the ideal of a perfectly parallel beam owing to the finite size of the light-radiant. The best $w \theta$ can do is to use as small a radiant as possible, and also as long a focus lens as we can without reducing the illumination too much. In the preliminary experiments a slit was used as the radiant, the light from a projection type of electric glow lamp being concentrated on it with a condensing lens. Between this slit and the mirror a long focus lens was placed, and the distance of the lens and the slit was so adjusted that the image of the latter was formed on the second slit behind which the eye was placed. The lens is naturally placed as close to the revolving mirror as possible, as the image formed will be magnified in the ratio of its distances from the two slits, while the speed of the image will be proportional to the distance of the mirror from what we might perhaps call the eye-slit ; thus the distance between the lens and the mirror only tends to enlarge the image without increasing the speed, and should hence be kept as small as is convenient. In the first trials this lens was of about 2 metres focal length, which was hardly sufficient. These experiments were conducted in an ordinary room, and it was found impossible to keep it sufficiently dark for the eye of the observer to become truly dark-adapted. The distance between the mirror and the eye-slit could only be made about 15 metres, a distance which it was thought could be increased with advantage.

On both these accounts it was decided to remove the apparatus to the cellars of this building. These cellars were particularly suitahle for the purpose, as a distance of 30 metres could easily be obtained between the mirror and the eye-slit, and no trouble was experienced in keeping them nearly perfectly dark. Some modifications were also introduced into the arrangements as a result of our previous trials. Thus we had already found that even at the maximum speed of the centrifuge the light was plainly visible in the mirror. This showed that a flash of the order of about $10^{-6}$ sec. or rather less was visible if repeated sufficiently often. To be able to deal with one flash of this duration, 
however, it is necessary to use some method of only illuminating the mirror at intervals for less than a complete revolution, thus ensuring that only one flash reached the eye for each light interval. This end was brought about by interposing a revolving disk, with a sector of suitable size cut out, between the lens and the radiant. 'The dimensions of the sector removed from the disk were arranged so that when the disk was running at about 120 r.p.m. the length of flash should be about $\frac{1}{150}$ sec., which would be less than one revolution of the centrifuge. The frequency of the flashes seen by the observer in this case was about two per second. To drive the disk, it was mounted directly on the shaft of a variable speed repulsion motor. As a matter of fact, in practice the disk was driven at a slightly greater speed than 120 r.p.m. 'This increased therfrequency of the flasbes slightly, but diminished the chance of any light from two successive revolutions of the mirror reaching the eye.

The optical arrangements were also improved. New lenses of various focal lengths were obtained from Adam Hilger \& Co. Finally a lens of about 4 metres focal length was found to answer most satisfactorily. If a longer focal length than this is used, the question of obtaining sufficient illumination in the beam becomes rather troublesome. Different forms of radiant were also tried. The plan of using a slit as radiant was abandoned as sufficient light could not be obtained from it. After trying various otter light sources, a small galvanometer electric lamp supplied by the Cambridge \& Paul Scientific Instrument Co. was found to work most satisfactorily. This lamp had a single loop filament, and by turning this loop parallel to the direction of the light-beam, a very fair approximation to a line source of light could be obtained.

The method of observing the flashes was as follows. One olserver took up his position at the eye-slit, which was fitted with a small tube so that the direction of vision might be preserved constant in the dark. The other observer then started the centrifuge and ran it up to the required speed. In the meanwhile the revolving sector shutter was kept permanently open, so that what the first observer perceived was a steady light in the mirror. When he had satisfied himself that this light was visible, i.e. that the direction of the light-beam was correct, he gave a sigual, and the sector shutter was started. This shutter was not, however, run continuously, but it was stopped in the open position occasionally, so that the observer might satisfy himself that the light-beam was reaching his eye correctly. This precaution 
is very necessary, owing to the fact that between the very feeble flashes that are visible when the revolving shrutter is running, there is a great tendency for the direction of the eye to alter, as in the dark intervals there is nothing to fix its position very exactly.

It is also very necessary that the dark adaptation of the eve should be nearly perfect. For this purpose the observer should be at least twenty minutes in the dark before attempting to make any trials. The difference in sensitivity between ordinary daylight vision and fully dark-adapted vision is very large. Selig Hecht, in a paper published in the 'General Journal of Physiology' for May 1920, states that the dark-adapted eye is easily from 5,000 to 10,000 times more sensitive. To attain the maximum sensitivity it is necessary to be at least 45 minutes in the dark, but after 25 minutes the increase in sensitivity is very slow, hence probably the period of 20 minutes adopted in these experiinents was sufficient to ensure that the eye would be nearly fully dark-adapted.

As regards the results obtained with this apparatus, it was found that a flash of $2 \times 10^{-7}$ sec. was still visible. A flash of duration $8 \times 10^{-8}$ sec. was, howrever, found to be invisible. This flash was, however, found to be visible if viewed directly without a slit, in which case the time of flash would be about $2.4 \times 10^{-7}$ sec., thus confirming the previous result. The energy in each of these flashes could be approximately calculated from the candle-power of the radiant and the varions dimensions of the apparatus, and it was found that, neglecting any loss by reflexion, etc., the energy in the visible flash was about $4 \times 10^{-7} \mathrm{erg}$, while that in the invisible flash was about $8 \times 10^{-8}$ erg. As to whether the invisibility of the shorter flash is due to its smaller duration alone or simpiy to the decrease in the energy available, is uncertain. It seems, however, that the latter hypothesis is more probable as the intensity of the longer flash was excessively feeble. The problem of getting more light into a flash of this nature is not very easy, as the amount of light which can be obtained depends only on the brightness of the radiant, and it is not very easy to get a suitable one which would be brighter than an electric light filament. An arc lamp might have given more light, but would nothave been so convenient as a slit, and a condensing lens would have been required. Probably the increase in brightness would also not have been very large.

Iveagh Geological Laboratory, Sept. 1921. 